\title{
Desarrollo y Reforma Educativa en Venezuela: El ensayo del Régimen semestre-crédito en educación media (1976- 1986) ${ }^{1}$
}

\author{
Development and educational reform in Venezuela: the attempt to \\ implement the semester-credit scheme in secondary education (1976-1986)
}

\begin{abstract}
Desenvolvimento e reforma educação na Venezuela: O regime semestre de teste na educação média (1976-1986)
\end{abstract}

Jadhiel Alejandro Rodríguez Suárez ${ }^{2}$

Universidad Centroccidental Lisandro Alvarado (Venezuela)

Recepción: 21/08/2016

Evaluación: 10/10/2017

Aceptación: 05/01/2018

Artículo de investigación - Reflexión

DOI: https://doi.org/10.19053/01227238.8149

\section{RESUMEN}

El presente artículo tiene el objetivo de reconstruir el proceso histórico del ensayo de régimen semestre-crédito en el subsistema de Educación Media del Sistema Educativo venezolano entre 1976 y 1986 en el marco de la Reforma Educativa; con el propósito de revelar elementos desconocidos o poco documentados de la Reforma Educativa venezolana y que tienen vinculación con la teoría desarrollista de la educación y su aplicación efectiva en la región latinoamericana. Siguiendo el método de la Historia Social como historia síntesis, historia total, se realizó una revisión amplia de fuentes documentales y bibliográficas con el propósito de describir el proceso histórico de la reforma educativa; analizar el proceso de planeación, lanzamiento, desarrollo y cancelación del ensayo de régimen semestre-crédito en el marco de dicha reforma; y valorar la relación entre Educación y Desarrollo en la historia contemporánea de Venezuela; llegándose a la conclusión que el ensayo régimen semestre-crédito, pese a sus ventajas, implicó la complejización de la administración escolar debido a su enfoque regionalista y descentralizado, por lo cual fue cancelado por la administración nacional a finales de la década de los ochenta del siglo XX.

Palabras clave: Revista Historia de la Educación Latinoamericana; historia de la educación media; reforma educativa; desarrollo.

1 Artículo de Reflexión elaborado como parte de la investigación para optar al Título de Magíster en Educación, mención Enseñanza de la Historia por la Universidad Pedagógica Experimental Libertador, Instituto Pedagógico de Barquisimeto "Luís Beltrán Prieto Figueroa" (UPEL-IPB) en Barquisimeto, Venezuela.

2 Magíster en Educación, mención Enseñanza de la Historia por la UPEL-IPB. Coordinador adjunto de la Cátedra Libre Historia de la Universidad perteneciente a la Universidad Centroccidental Lisandro Alvarado (UCLA). Estudiante del Doctorado en Cultura Latinoamericana y Caribeña en la UPEL-IPB. Correo electrónico: jadhielalers@gmail.com. 


\section{ABSTRACT}

This paper aims to reconstruct the historical process of the semester-credit regime attmept in the Venezuelan subsystem of Secondary Education between 1976 and 1986 within the framework of the Educational Reform. Here are also revealed unknown or little documented elements related to the developmentalist theory of education and its effective application in the Latin American region. By adapting the method of Social History as synthesis history, total history, a wide review of documentary and bibliographical sources was carried out with the purpose of describing the historical process of educational reform; analyzing the process of planning, implemantation, development and cancellation of the semester-credit scheme attempt within the framework of this reform. The relationship between Education and Development in the contemporary history of Venezuela was finally assessed and it was possible to conclude that the semester-credit system attempt, despite its advantages, led to complicate the school administration because of its regionalist and decentralized nature, and precisely for this reason, it was canceled by the national administration in the late eighties of the twentieth century.

Keywords: Journal History of Latin American Education; history of secondary education; education reform; development.

\section{RESUMO}

O presente artigo tem como objetivo reconstruir o processo histórico do teste de regime de crédito semestral no subsistema de ensino médio do sistema educacional venezuelano entre 1976 e 1986 no âmbito da reforma educacional; com o objetivo de revelar elementos documentados desconhecidos ou desconhecidos da Reforma Educacional venezuelana e que têm vínculos com a teoria desenvolvimentista da educação e sua aplicação efetiva na região latino-americana. Seguindo o método da História Social como história de síntese, história total, uma ampla revisão de fontes documentais e bibliográficas foi realizada com o objetivo de descrever o processo histórico de reforma educacional; analisar o processo de planejamento, lançamento, desenvolvimento e cancelamento do julgamento do regime de crédito semestral no âmbito da referida reforma; e avaliar a relação entre Educação e Desenvolvimento na história contemporânea da Venezuela; Concluiu-se que o esquema de crédito semestral, apesar de suas vantagens, envolveu a complexidade da administração escolar devido à sua abordagem regionalista e descentralizada, para a qual foi cancelada pela administração nacional no final da década de 1980. Século XX.

Palavras-chave: Revista de História da Educação Latino-americana; história da educação secundária; reforma educacional; desenvolvimento.

\section{INTRODUCCIÓN}

Los procesos de reforma educativa en Latinoamérica durante la segunda mitad del siglo XX estuvieron signados por las ideas hegemónicas a nivel hemisférico, de transformación de los Sistemas Escolares en función del desarrollo social y económico.

Para lograr este propósito, los Estados latinoamericanos recurrieron a la cooperación internacional tanto para capacitar a su personal docente y administrativo, como para el asesoramiento técnico de los procesos de reforma que se planificaron y aplicaron. Este doble proceso de apropiación devino en 
la implantación de modelos educativos no tradicionales, con la esperanza de transformar las sociedades y economías de Latinoamérica al Desarrollo.

En el caso de Venezuela, la Reforma Educativa comenzó a planificarse desde 1958, pero no sería sino hasta 1969 que sería aplicada. El desarrollo histórico de dicha reforma involucró pues, interesantes ensayos como el régimen semestrecrédito: un ensayo para la semestralización de la educación media en el país.

Y es que el Desarrollo como propósito y meta de la sociedad ha sido el leitmotiv de la Educación en los países del llamado Tercer Mundo, en especial en Latinoamérica; y la Reforma Educativa su expresión más característica.

El caso que se describe y analiza en el presente artículo se enmarca dentro de los esfuerzos llevados adelante por el Estado venezolano para alcanzar el tan ansiado Desarrollo económico y social, utilizando el proceso de Reforma Educativa como contexto y pretexto para experimentar estrategias y enfoques.

En efecto, el ensayo de régimen semestre-crédito fue un proyecto educativo implementado en el subsistema de Educación media diversificada de Venezuela durante el periodo 1976-1986 en treinta y dos institutos piloto.

Propuesto en 1976 como un salto hacia adelante en el proceso de Reforma Educativa iniciado en 1969, el ensayo de régimen semestre-crédito involucró no solo la semestralización del régimen escolar, sino la aplicación de estrategias y planes de estudios ajustados al paradigma del Desarrollo regional como ruta para la diversificación geoeconómica para, posteriormente, lograr el despegue de las fuerzas productivas y por ende el Desarrollo económico y social.

Pese a la riqueza teórica del proyecto del ensayo de régimen semestre-crédito y lo que representó durante más de una década en Venezuela, muy poca literatura especializada hace referencia al tema, posiblemente por el poco impacto que tuvo en el desarrollo del Sistema Educativo. Sin embargo, en los institutos en los que se aplicó el ensayo, se pudo rastrear la trascendencia que tuvo el plan de estudios y las estrategias didácticas contempladas en el proyecto.

Es así como se llegó al hallazgo del ensayo de régimen semestre-crédito: gracias a las pesquisas sobre la Historia Social de la Educación Media en Venezuela, en las que se reflejó el impacto que tuvieron los planes de estudio, las estrategias didácticas, y el régimen escolar en el desempeño posterior de los egresados de institutos piloto del mencionado ensayo, como el Liceo "Rafael Villavicencio" de Barquisimeto.

Dichas pesquisas y el presente artículo mismo, se elaboraron siguiendo el método de la Historia Social, historia síntesis o historia total. Enfoque teóricometodológico formulado por la Escuela francesa de Annales y que encontró eco en Venezuela en los estudios del Dr. Federico Brito Figueroa y los de su discípulo, el Dr. Reinaldo Rojas, quien dirige la Escuela de Historiadores de Barquisimeto; escuela prolífica en estudios de historia de la educación. Este enfoque busca aprehender la totalidad del hecho histórico considerando los elementos sociales, económicos, políticos y culturales a fin de lograr una visión síntesis de la realidad: compleja y multidimensional. 


\section{Educación para el Desarrollo}

Durante la década de los años cincuenta del siglo XX se asistió a la formulación teórica del concepto de modernización ${ }^{3}$ como correlato de la industrialización, esto es, el proceso previo al desarrollo nacional ${ }^{4}$.

Según esta teoría, los países no-industrializados debían, intencionalmente, encaminar sus esfuerzos hacia la modernización absoluta de todos los elementos sociales de la nación, es decir, plantearse una transición desde un estadio caracterizado por elementos pre-capitalistas, preindustriales, rurales y religiosos, hacia uno capitalista, industrial, urbano y secular. Esta modernización debía venir aparejada al proceso de desarrollo nacional, es decir, un conjunto de acciones que incluían la redistribución del ingreso (fomento del consumo masivo), del poder político (democratización progresiva), de las actividades económicas (industrialización) y de la cultura (alfabetización y educación gratuita). ${ }^{5}$

El Estado venezolano que recién (enero de 1958) había reiniciado su proceso de democratización asumió este enfoque como parte fundamental de las reformas que había emprendido. ${ }^{6}$

Así pues, este proceso condujo a la aparición de dos rasgos importantes en la cultura educativa venezolana: en primer lugar, "la adquisición de una cultura técnica, de modos de pensar, hacer y resolver los problemas de la educación, no siempre convenientes para las condiciones del país"; y en segundo lugar "la reproducción acrítica de modelos poco exitosos en nuestro medio" con el consecuente desprecio a las tradiciones pedagógicas venezolanas. ${ }^{7}$

Esta idea encontraba eco a nivel internacional.

Desde finales de la década de los cincuenta, numerosos académicos norteamericanos como Theodore W. Schultz promovieron la idea de la necesaria inversión en educación para lograr el desarrollo económico, con lo que las agencias creadas para la asistencia económica en la postguerra ${ }^{8}$ se dedicaron a la asistencia educacional justificándose tanto en el deseo de aumentar las ganancias para los inversionistas norteamericanos al capacitar mano de obra calificada, como en un concepto de seguridad nacional norteamericana al asegurarse la alianza de las naciones periféricas ${ }^{9}$.

Es en este contexto que se crea, recién instaurado el Estado Democrático en Venezuela, la Oficina de Planeamiento Integral de la Educación (EDUPLAN)

3 Carlos Altamirano. “Modernización”, en Diccionario de Ciencias Sociales y Políticas, ed. Torcuato S. Di Tella. (Buenos Aires: Puntosur Editores, 1989), 397-398.

4 Pedro Paz. "Desarrollismo" y "Desarrollo-Subdesarrollo", en Diccionario de Ciencias Sociales y Políticas, ed. Torcuato S. Di Tella. (Buenos Aires: Puntosur Editores, 1989), 168-170.

5 Theotonio Dos Santos. "La Crisis de la Teoría del Desarrollo y las relaciones de dependencia en América Latina”, en La dependencia político-económica de América Latina, eds. Helio Jaguaribe. (México: Siglo XXI Editores, 1978) ,160-161.

6 Alejandro Bilbao y Carmen Gibaja. "Legislación educativa y dependencia en Venezuela", Cuadernos de Educación, № 38 (1976): 45-46.

7 Nacarid Rodríguez. Historia de la Educación Venezolana. Seis ensayos. (Caracas: Universidad Central de Venezuela, 1996), $243-244$.

8 Banco Internacional de Reconstrucción y Fomento (BIRF) que luego pasará a llamarse Banco Mundial, y la Agencia Internacional para el Desarrollo (AID)

9 Martin Carnoy. La Educación como Imperialismo Cultural. (México, Siglo XXI Editores, 1988), 300-301 
el 07 de agosto de 1959. El Estado venezolano precisaba de un organismo capaz de dirigir y controlar el proceso de planeamiento de la educación según los estándares internacionales si aspiraba a beneficiarse de los convenios con las fundaciones estadounidenses. Los agentes intermediarios de este proceso fueron la UNESCO y la CEPAL. De modo que EDUPLAN funcionó como el agente de enlace entre los organismos internacionales y el sistema educativo con atribuciones para planear, coordinar, asesorar, orientar, supervisar y evaluar todos los planes, programas y proyectos emprendidos por el Ministerio de Educación.

Este complejo esfuerzo estatal dirigido desde EDUPLAN, de preparar técnica, científica y racionalmente la futura reforma educativa, demuestra además el nivel de participación que tenían los organismos internacionales en este proceso. ${ }^{10}$

En este sentido, fue emblemático el convenio entre el Estado venezolano y la Universidad de Wisconsin a través de la Fundación Ford en julio de 1967 especificando la participación de técnicos y especialistas de la mencionada universidad en el mejoramiento de "los planes educativos en Secundaria, para hacerlos más cónsonos con el desarrollo económico que se opera en el país". ${ }^{11}$

Al iniciar la década de los setenta, el Estado venezolano va a acelerar y profundizar este proceso. Dos factores incidieron en ello, ambos enmarcados en la bonanza económica producto de la inusitada alza de los precios del petróleo: a) el impulso dado por el Estado venezolano a la industrialización a través de mayores inversiones, y la nacionalización de las industrias minero-extractivas del hierro y los hidrocarburos; y b) la profundización de los problemas estructurales de la economía rentista. Esto tanto en el campo económico como en el campo cultural. ${ }^{12}$

En ese sentido, el IV Plan de la Nación correspondiente al periodo 19701975 estableció como objetivo fundamental de la educación "la conformación de una sociedad que se realice dentro de un proceso de desarrollo sostenido y autónomo"13, esto es, la educación como "Empresa Nacional". ${ }^{14}$

Esta concepción de la modernización permaneció en la esencia del V Plan de la Nación elaborado para el periodo 1975-1980.

\section{La Reforma Educativa y Educación Media Diversificada}

Tanto el V Plan de la Nación como su predecesor implementaron la misma idea de modernización y desarrollo: acelerar el surgimiento de un capitalismo

10 Rodríguez, Historia, 244.

11 Simón Sáez Mérida, “Crédito educativo y modelo tecnocrático", Cuadernos de Educación, Número extraordinario 63-64. (1979): 31. Este convenio fue ratificado en 1972 por cinco (05) años más hasta 1977.

12 Diferentes autores entre los que destacan Mattelart, Vasconi, Selser, Swchebor y Carnoy han dado cuenta de un proyecto educativo estadounidense para América Latina caracterizado por la estandarización de técnicas, procedimientos y fines.

13 Omar Belandria y Ricardo H. Velarde, "Segunda hipótesis para un estudio del sistema escolar en Venezuela", Cuadernos de Educación, Número extraordinario 44-45 (1977): 139.

14 Manuel Fermín, Momentos históricos de la Educación Venezolana. (Caracas: Editorial Romor, 1989), 104. 
nacional independiente apoyado en una masa de trabajadores técnicamente calificados, y en un sector de profesionales e investigadores de alto nivel.

De esta forma, la administración del presidente Rafael Caldera (1969-1974) comenzó emitiendo 11 decretos y 18 resoluciones dirigidas a regular el sistema educativo. Entre ellos el de mayor significación -decreto №. 120, del 13 de agosto de 1969- adoptó el proyecto ensayado por EDUPLAN desde 1963 de dividir la Educación Media en dos ciclos: un Ciclo Básico Común, de tres años de duración, obligatorio para todas las ramas de la Educación Media, y un Ciclo Diversificado, de dos años como mínimo.

Así pues, los grandes liceos nacionales que otrora agruparan todos los niveles de la Educación Media y que habían desempeñado un rol fundamental en la vida política del país, fueron reestructurados. Entre 1969 y 1975 la mayoría pasaron a constituirse como Ciclos Diversificados impartiendo solo dos años de escolaridad, reduciendo con ello el tiempo de convivencia del estudiantado venezolano con el resto de los elementos de la comunidad educativa.

De igual manera, en el sector universitario, la Reforma introdujo la semestralización como parte del proceso de modernización de las universidades e institutos de educación superior. Semestralización que a pesar de sus detractores ${ }^{15}$, perseguía el mismo fin desarrollista expresado en las reformas a la educación media.

Esta intencionalidad desarrollista se encuentra expresada en los textos de los decretos Nos. 120 y 136 que justifican la Reforma por la necesidad de formar "recursos humanos aptos para la promoción del desarrollo integral de la República y de cada una de sus regiones según las peculiaridades culturales, económicas y sociales"16, además de hacerlo en base a las políticas establecidas en el Plan Nacional de Desarrollo y en "atención a las peculiaridades y grado de desarrollo de las regiones de la República sin perjuicio de su unidad".

El segundo de los propósitos señalados en los documentos de la Reforma Educativa, se traducía en la regionalización de la educación, es decir, el esfuerzo de desconcentración y descentralización de los procesos administrativos y académicos, a fin de que los planteles de Ciclo Diversificado se convirtieran en centros de gestión del desarrollo regional preparando simultáneamente a la población escolar para proseguir hacia la educación superior, si fuese el caso, y para incorporarse al mercado de trabajo si fuese necesario. ${ }^{17}$

En este sentido, es emblemática la respuesta del Prof. Alberto Armitano, Director de Control y Evaluación de Estudios del Despacho del Ministerio de Educación para la época, al ser interrogado sobre el propósito de la Educación Diversificada: "Primero: lograr una educación diferenciada que responda a la geografía del país, a sus recursos y sus necesidades. Segundo: adaptar la

\footnotetext{
15 Rodríguez, Historia, 264.

16 Presidencia de la República. "Decreto No 120: Reglamento para la Educación Secundaria y la Educación Técnica", Gaceta Oficial de la República de Venezuela, Caracas, 19 de agosto de 1969

17 Ministerio de Educación. Eduplan informa, vol. 5 № 4 (1972): 35.
} 
educación al medio (...) Tercero: esta educación que se dé a nuestros estudiantes, debe contribuir al desarrollo regional". ${ }^{18}$

Y es que según los expertos del Ministerio de Educación “Todo el talento venezolano se concentra en la capital. La provincia es continuamente abandonada en cuanto se adquiere alguna capacitación", por lo que el Ciclo Diversificado debía coadyuvar para que "el estudiante preparado en una región del país, de acuerdo a la demanda local de empleo, permanezca allí brindando sus servicios y conocimientos y favoreciendo con ello el desarrollo de esa región" ${ }^{19}$

Es por esta razón que el plan de estudios del Ciclo Diversificado se diseñó con la específica función de contribuir al desarrollo de las regiones venezolanas, distribuyendo las especialidades y menciones según las necesidades y potencialidades detectadas en estudios y diagnósticos previos. ${ }^{20}$

No es atrevido afirmar, que el desarrollo regional fue el leitmotiv de la Educación Diversificada en Venezuela, por lo menos en sus orígenes.

En el campo de la Educación, este modelo de desarrollo se dirigió inicialmente a las Universidades consideradas centros de gestión del desarrollo regional por sus potencialidades de capacitación en innovación. ${ }^{21}$

El aporte fundamental de la Reforma Educativa fue haber extendido este concepto a la Educación Media a través del diseño regionalizado del Ciclo Diversificado.

\section{La Reforma Educativa y el ensayo del régimen semestre-crédito}

Ese propósito pareció lograrse en la segunda mitad de la década de los setenta, cuando el alza inesperada de los precios del petróleo permitió a la administración del presidente Carlos Andrés Pérez (1974-1979) profundizar la Reforma Educativa y extenderla con proyectos de modernización más audaces, en especial la semestralización a través de la introducción del denominado Régimen Semestre-Crédito: una adaptación del sistema de créditos curriculares recién aplicado en las universidades.

El ministro de Educación Luis Manuel Peñalver, su más entusiasta promotor ${ }^{22}$, informaba en su intervención ante el Congreso Nacional en 1976 sobre la implementación del Régimen en diez (10) instituciones de Educación Media del país anunciando "instrumentalizar e implantar el régimen de semestralización en 32 planteles de Educación Media." 23 Sin embargo, en marzo de 1977 reconoció las dificultades que semejantes modelos educativos presentaban para su implementación inmediata, y la decisión de aplicarlos en forma experimental

\footnotetext{
18 Ibíd, 39.

19 Ibíd, 39.

20 Ibíd, 04-05.

21 Reinaldo Rojas. Universidad Centroccidental Lisandro Alvarado: una historia de 50 años. (Barquisimeto: Universidad Centroccidental Lisandro Alvarado, 2012), 59-60.

22 Ministerio de Educación. Memoria y Cuenta de 1975. (Caracas: Ministerio de Educación, 1976), XLVII.

23 Ibíd, LXII
} 
hasta determinar las formas más convenientes de adaptarlas a la realidad educativa del país ${ }^{24}$, planteando como fecha tentativa para su evaluación el año escolar 1977-1978. ${ }^{25}$

Según el proyecto del Ministerio de Educación, el ensayo del régimen semestre-crédito sería implementado en dos etapas diferentes ${ }^{26}$ : la primera, a partir de enero de 1976, incluiría a los liceos: "Francisco de Miranda" (Los Teques, Estado Miranda), "Combinado Valencia” (Valencia, Estado Carabobo), "Carmelo Fernández" (Guama, Estado Yaracuy), "Udón Pérez" (Maracaibo, Estado Zulia), “Don Ramón Velásquez” (San Cristóbal, Estado Táchira), “Carlos Francisco Grisanti" (Río Caribe, Estado Sucre), "Francisco Lazo Martí" (San Fernando de Apure, Estado Apure), "Ramón Bastidas" (Turmero, Estado Aragua), "Jacinto Lara" (Cabudare, Estado Lara) ${ }^{27}$

Mientras que la segunda etapa iniciaría en el mes de enero de 1977 e incluiría a los liceos: "Julián Maza" (Ciudad Bolívar, Estado Bolívar), "Aplicación" (Caracas, Distrito Federal), "Augusto Pi Suñer" (El Junquito, Distrito Federal), "Esteban Smith" (Coro, Estado Falcón), "Agustín Aveledo" (Caracas, Distrito Federal), "Rafael Villavicencio" (Barquisimeto, Estado Lara), "Pedro Arnal" (Cumaná, Estado Sucre), "Dr. Felipe Guevara Rojas" (Cantaura, Estado Anzoátegui), “Félix Ángel Lozada” (Maturín, Estado Monagas), “José Antonio Anzoátegui" (Barcelona, Estado Anzoátegui), "Alejandro Humboldt" (Calabozo, Estado Guárico), "Carlos Rangel-Lamus" (Rubio, Estado Táchira), "Rómulo Gallegos" (Maracaibo, Estado Zulia), "Emiro Fuenmayor" (Betijoque, Estado Trujillo), "Fray Ramos de Lora" (Mérida, Estado Mérida), "Nueva Esparta" (Porlamar, Estado Nueva Esparta), "José Vicente de Unda" (Guanare, Estado Portuguesa), "Alberto Arvelo Torrealba" (Barinas, Estado Barinas), "José Luis Ramos" (Maracay, Estado Aragua), "Enrique Bernardo Núñez" (Valencia, Estado Carabobo), "Diversificado San Carlos" (San Carlos, Estado Cojedes), "Pérez Bonalde" (Ocumare del Tuy, Estado Miranda), "Rómulo Gallegos" (San Felipe, Estado Yaracuy), y "Yaritagua" (Yaritagua, Estado Yaracuy) ${ }^{28}$

Las herramientas de este nuevo enfoque/estrategia fueron, en síntesis, la semestralización y la individualización de los estudios de Educación Media.

En cuanto a la semestralización, el régimen semestre-crédito estuvo diseñado para cursar la Educación Media Diversificada en cuatro (04) semestres -en sustitución de los dos (02) años del régimen tradicional- con una duración mínima de dieciocho (18) semanas cada uno, de las cuales dieciséis (16) semanas serían para las actividades pedagógicas, y dos (02) semanas para evaluación y eventual recuperación. Entre cada semestre se previó un periodo inter-semestral

24 Sáez Mérida, Crédito, 62-68.

25 Ministerio de Educación. Memoria y Cuenta de 1976. (Caracas, Ministerio de Educación, 1977), XXIV.

26 Ibíd, 130-131.

27 Ministerio de Educación. "Resolución N 56 del 07 de abril de 1976”, Gaceta Oficial de la República de Venezuela, Caracas, 22 de abril de 1976.

28 Ministerio de Educación. "Resolución N 12 del 05 de enero de 1977", Gaceta Oficial de la República de Venezuela, Caracas, 11 de enero de 1977. Hay incongruencias en las cifras presentadas por el Ministerio de Educación en diferentes documentos. Ver: Ministerio, Memoria 1976, 130-131. 
a fin de efectuar las inscripciones de las asignaturas, mismas que tenían un valor en términos de créditos. ${ }^{29}$

Debido a la relativa complejidad de este régimen en comparación con el tradicional régimen anual, por cada treinta (30) alumnos los planteles experimentales designaban un profesor asesor con el propósito de orientar académica y profesionalmente a los estudiantes. De igual manera, asignaban profesores tutores a cada estudiante para prestar asistencia y apoyo necesario en situaciones de recuperación y re-enseñanza. ${ }^{30}$

En cuanto a la individualización, se trataba de proporcionar al alumno las condiciones para que la progresión de sus estudios dependiera exclusivamente de sus habilidades e intereses.

En efecto, la aplicación del ensayo significó, en algunos casos, un comportamiento errático en la matrícula de los planteles experimentales debido a la individualización que el nuevo régimen permitía al estudiantado, en especial en lo concerniente al adelanto de asignaturas por medio de tutorías y pruebas especiales, por lo que la matrícula fluctuaba dependiendo de las características de cada generación de estudiantes. Este comportamiento era dinamizado por la estructura misma del plan de estudios del Régimen.

En efecto, la formación del alumno abarcaba cuatro (04) grandes áreas:

La correspondiente a un área común, constituida por un conjunto de asignaturas de carácter obligatorio, "necesarias para complementar el proceso de integración a la cultura general", iniciado en el CBC. La segunda se trataba de un área diversificada, integrada por asignaturas específicas de cada mención, destinadas a "proporcionar al estudiante los elementos teórico-prácticos para la continuación de sus estudios profesionales, según la mención seleccionada".

A estas áreas se le añadían un área electiva, constituida por asignaturas de libre elección con el objetivo de lograr que los alumnos, de acuerdo a sus intereses y capacidades, profundizaran y ampliaran "su formación en el estudio de los diferentes campos del conocimiento científico, tecnológico, y humanístico"; así como también un área exploratoria y extra-académica (A.E.E.) compuesta por un conjunto de actividades orientadas a planificar el tiempo libre de los alumnos. ${ }^{31}$

\section{Cuadro I}

Plan de estudios del Ciclo Diversificado de Educación Secundaria bajo el Ensayo del Régimen Semestre-Crédito

\begin{tabular}{l|l|l|l}
\hline \multicolumn{3}{c|}{ Ádigo } & \multicolumn{1}{c}{ Asignatura } \\
\hline LL & 1103 & Lengua y Literatura I & \multicolumn{1}{c}{ Pre-requisitos } \\
\hline LL & 1203 & Lengua y Literatura II & NO TIENE \\
\hline MAT & 1103 & Matemática I & NO TIENE \\
\hline MAT & 1203 & Matemática II & MAT 1103 \\
\hline
\end{tabular}

29 Ministerio de Educación. Guía del Estudiante. Régimen de Semestre-Crédito para los Institutos Experimentales de Educación Media Diversificada. Menciones de Humanidades y Ciencias. (Caracas: Ministerio de Educación, 1975), 08.

30 Ibíd, 06

31 Ministerio, Memoria 1976, 90. 


\begin{tabular}{l|l|l|l}
\hline CS & 2113 & Historia Contemporánea de Venezuela I & NO TIENE \\
\hline CS & 2213 & Historia Contemporánea de Venezuela II & CS 2113 \\
\hline CS & 2123 & Geografía Económica de Venezuela I & NO TIENE \\
\hline CS & 2223 & Geografía Económica de Venezuela II & CS 2123 \\
\hline ID & 1123 & Inglés I & NO TIENE \\
\hline ID & 1223 & Inglés II & ID 1123 \\
\hline EF & 1101 & Educación Física I & NO TIENE \\
\hline EF & 1201 & Educación Física II & EF 1101 \\
\hline EF & 2101 & Educación Física III & EF 1201 \\
\hline EF & 2201 & Educación Física IV & EF 2101 \\
\hline
\end{tabular}

\section{ÁREA DIVERSIFICADA}

\begin{tabular}{|c|c|c|c|}
\hline \multicolumn{4}{|c|}{ Mención Ciencias } \\
\hline & Código & Asignatura & Pre-requisitos \\
\hline LL & 2113 & Lengua y Literatura III & LL 1203 \\
\hline FIS & 1113 & Física I & NO TIENE \\
\hline FIS & 1213 & Física II & FIS 1113 \\
\hline QUI & 1123 & Química I & NO TIENE \\
\hline QUI & 1223 & Química II & QUI 1123 \\
\hline $\mathrm{BIO}$ & 2113 & Biología I & QUI 1223 \\
\hline $\mathrm{BIO}$ & 2213 & Biología II & BIO 2113 \\
\hline MAT & 2103 & Matemática III & MAT 1203 \\
\hline MAT & 2203 & Matemática IV & MAT 2103 \\
\hline ART & 1121 & Dibujo I & NO TIENE \\
\hline ART & 1221 & Dibujo II & ART 1121 \\
\hline \multicolumn{4}{|c|}{ Mención Humanidades } \\
\hline & Código & Asignatura & Pre-requisitos \\
\hline $\mathrm{CS}$ & 1133 & Sociología I & NO TIENE \\
\hline CS & 1233 & Sociología II & CS 1133 \\
\hline CS & 1103 & Filosofía I & NO TIENE \\
\hline $\mathrm{CS}$ & 1203 & Filosofía II & CS 1103 \\
\hline MAT & 2133 & Matemática III & MAT 1203 \\
\hline MAT & 2233 & Matemática IV & MAT 2133 \\
\hline ART & 2133 & Historia del Arte I & NO TIENE \\
\hline ART & 2233 & Historia del Arte II & ART 2133 \\
\hline LL & 2133 & Lengua y Literatura III & LL 1203 \\
\hline LL & 2233 & Lengua y Literatura IV & LL 2133 \\
\hline
\end{tabular}

ÁREA ELECTIVA

Semestres I y II para ambas menciones

\begin{tabular}{l|l|l|l}
\hline \multicolumn{2}{c|}{ Código } & \multicolumn{1}{c|}{ Asignatura } & \multicolumn{1}{c}{ Pre-requisitos } \\
\hline ART & 1112 & Artes Plásticas I & NO TIENE \\
\hline ART & 1212 & Artes Plásticas II & ART 1112 \\
\hline CS & 1102 & Psicología I & NO TIENE \\
\hline CS & 1202 & Psicología II & CS 1102 \\
\hline ID & 1102 & Latín y griego I & NO TIENE \\
\hline
\end{tabular}


Régimen semestre-crédito en educación media (1976-1986)

\begin{tabular}{|c|c|c|c|}
\hline ID & 1202 & Latín y griego II & ID 1102 \\
\hline LOG & 1122 & Lógica & NO TIENE \\
\hline FIS & 1122 & Ciencias de la Tierra I & NO TIENE \\
\hline FIS & 1232 & Ciencias de la Tierra II & FIS 1122 \\
\hline ART & 2162 & Historia de la Cultura & NO TIENE \\
\hline \multicolumn{4}{|c|}{ Semestres III y IV para ambas menciones } \\
\hline \multicolumn{2}{|r|}{ Código } & \multirow{2}{*}{\begin{tabular}{|c|} 
Asignatura \\
Seminario sobre Problemática Petrolera
\end{tabular}} & Pre-requisitos \\
\hline CS & 2173 & & NO TIENE \\
\hline $\mathrm{CS}$ & 2173 & Seminario sobre Problemática Siderúrgica & NO TIENE \\
\hline CS & 2193 & Seminario sobre Educación Sexual & NO TIENE \\
\hline CS & 2133 & Seminario sobre Desarrollo Regional & NO TIENE \\
\hline CS & 2273 & $\begin{array}{l}\text { Seminario sobre Problemática del Agro } \\
\text { venezolano }\end{array}$ & NO TIENE \\
\hline CS & 2163 & $\begin{array}{|lll|}\begin{array}{l}\text { Seminario sobre la Integración } \\
\text { Latinoamericana }\end{array} & & \\
\end{array}$ & NO TIENE \\
\hline ID & 2163 & Francés I & NO TIENE \\
\hline ID & 2263 & Francés II & ID 2163 \\
\hline QUI & 2143 & $\begin{array}{l}\text { Química III (02 horas teóricas y } 03 \text { horas } \\
\text { prácticas) }\end{array}$ & QUI 1123 \\
\hline QUI & 2243 & Química IV & QUI 1223 \\
\hline FIS & 2153 & $\begin{array}{l}\text { Física III (02 horas teóricas y } 03 \text { horas } \\
\text { prácticas) }\end{array}$ & FIS 1113 \\
\hline FIS & 2253 & $\begin{array}{l}\text { Física IV (02 horas teóricas y } 03 \text { horas } \\
\text { prácticas) }\end{array}$ & QUI 1223 \\
\hline \multicolumn{4}{|c|}{ ÁREA EXPLORATORIA Y EXTRA-ACADÉMICA } \\
\hline \multicolumn{2}{|r|}{ Código } & Asignatura & Pre-requisitos \\
\hline AEE & 1101 & $\begin{array}{|lll|}\begin{array}{l}\text { Actividades exploratorias } \\
\text { académicas I }\end{array} & y \quad \text { extra- } \\
\end{array}$ & NO TIENE \\
\hline AEE & 1201 & $\begin{array}{|llll|}\begin{array}{l}\text { Actividades exploratorias } \\
\text { académicas II }\end{array} & y \quad \text { extra- } \\
\end{array}$ & NO TIENE \\
\hline AEE & 2101 & $\begin{array}{|lll|}\begin{array}{l}\text { Actividades exploratorias } \\
\text { académicas III }\end{array} & y \quad \text { extra- } \\
\end{array}$ & NO TIENE \\
\hline AEE & 2201 & $\begin{array}{l}\begin{array}{l}\text { Actividades exploratorias } \\
\text { académicas IV }\end{array} \\
\end{array}$ & NO TIENE \\
\hline
\end{tabular}

FUENTE: Ministerio de Educación, Ensayo Régimen Semestre Crédito: Una nueva estrategia y un nuevo enfoque para la educación diversificada. (Caracas: Ministerio de Educación, 1977), 10-13

Elaborado por: Jadhiel Rodríguez Suárez.

Es importante añadir que además de las asignaturas electivas, las instituciones podían ofrecer otras de acuerdo a sus recursos, y sobre todo y más importante, a las necesidades y potencialidades de la región donde estuvieran ubicadas ${ }^{32}$, siempre y cuando recibieran la aprobación correspondiente de la Dirección de Educación Media del Ministerio de Educación.

32 Destaca la asignatura Desarrollo regional en el Plan de estudios del Régimen, junto a seminarios sobre el desarrollo de sectores estratégicos del país como la industria siderúrgica y la industria petrolera. 
Así como en lo concerniente al plan de estudios el régimen de evaluación y sus prácticas correspondientes presentaba importantes diferencias con el tradicional régimen anual.

En primer lugar, la "expresión e interpretación de los resultados de la actuación del alumno", comenzó a realizarse mediante el uso de dos (02) escalas de calificación: una numérica, del uno (01) al nueve (09) para "aquellos aspectos cuantificables" del proceso de evaluación, con un mínimo aprobatorio de cuatro (04) puntos; y una escala literal de tres valores (A, B y C) para los "rasgos no cuantificables, con fines de descripción" ${ }^{33}$ entre excelente (A) y deficiente (C).

Sin embargo, la diferencia más trascendental con respecto a los regímenes de evaluación precedentes y paralelos era la posibilidad de acelerar el cumplimiento de la escolaridad a través de la aplicación de pruebas especiales, así como también la posibilidad de aprobar asignaturas pendientes de semestres anteriores, mientras el estudiante continuaba avanzando en otras asignaturas. ${ }^{34}$

Para asegurarse de que el número de estudiantes que requirieran aprobar asignaturas por vía extraordinaria, los diseñadores del ensayo de régimen semestre-crédito incorporaron la caracterización de diversas técnicas de enseñanza-aprendizaje en los manuales dirigidos a los docentes ${ }^{35}$. Es importante destacar que estas técnicas solo fueron institucionalizadas en el régimen anual con la promulgación del Reglamento General de 1986.

\section{Cancelación del ensayo de régimen semestre-crédito}

No obstante las múltiples ventajas del Régimen, existían serias dificultades que impedían el despliegue total del proyecto. Se trataba de dificultades inherentes a la centralización de los procesos administrativos que experimentaba el Sistema Educativo desde su creación a pesar de los esfuerzos de regionalización, desconcentración y descentralización, que redundaba en la desidia y el abandono de planes, programas y proyectos. ${ }^{36}$

En este sentido, el progresivo abandono por parte del Estado venezolano del ensayo llegó a su punto culminante con la Reforma Curricular llevada adelante por la administración del presidente Jaime Lusinchi (1984-1989) que incluyó la promulgación del Reglamento General de la Ley Orgánica de Educación en 1986, que imponía detalladas directrices al proceso de evaluación escolar; en especial la Resolución $\mathrm{N}^{\circ} 140$ contentiva de las Regulaciones Complementarias sobre el proceso de evaluación en los niveles de Educación Preescolar, Básica, y Media Diversificada y

35 "Estudio dirigido, Tarea dirigida, Interrogatorio, Debate, Mesa redonda, Simposio, Foro, Panel y Phillips 66". Ministerio, Memoria 1976, 32-37.

36 El ministro Luis Manuel Peñalver, principal promotor del ensayo del régimen semestre-crédito, fue destituido en 1977 con lo que el proyecto de semestralización en Educación Media empezó a decaer en cuanto al apoyo institucional. Fermín, Momentos, 119-120. 
Profesional ${ }^{37}$. Los planteles bajo ensayo de régimen semestre-crédito entraron así en un limbo jurídico sin precedentes.

En vista de ello, el 04 de mayo de 1987 el Ministerio de Educación decidió reformar la Resolución $\mathrm{N}^{\circ} 140$ modificando el contenido del Artículo $45^{\circ}$ con la incorporación de un ultimátum a los planteles bajo ensayo de régimen semestre-crédito de "acogerse al presente régimen de evaluación o continuar con el que habían venido aplicando... hasta tanto se implante el nuevo diseño curricular para el nivel de Educación Media Diversificada y Profesional" cuando procederían entonces a aplicar definitivamente lo establecido en la legislación educativa vigente para el momento. ${ }^{38}$

La respuesta de los planteles experimentales fue retornar inmediatamente al régimen anual antes que la reconversión se realizase de forma brusca por parte del Ministerio de Educación.

Ahora bien, no es coincidencia que la interrupción del ensayo ocurriese en medio de la crisis económica de finales de la década de los ochenta.

La recesión económica iniciada en 1978, recrudeció a partir de 1983 con el inicio de la escalada devaluativa de la moneda nacional ${ }^{39}$. La crisis impulsó a la sociedad venezolana a un debate nacional sobre las causas del desastre económico y las soluciones más viables para salir de la misma, desembocando así en una unánime exigencia al Estado para reformar el Sistema Educativo para hacerlo más cónsono con las necesidades productivas del país.

La administración del presidente Luis Herrera Campins (1979-1984) inició así un proceso de revisión de los planes, programas y proyectos en todos los sectores de la administración pública, incluyendo el ensayo del régimen semestre-crédito en la Educación Media Diversificada. ${ }^{40}$

A pesar de los comentarios favorables que se le hizo a su plan de estudios, al rol propedéutico que significaba para los aspirantes a cursar la Educación Superior, y a la importancia que se le daba a las actividades extra-académicas; en estos estudios se descubrió que a pesar de los esfuerzos emprendidos por docentes y directivos, la complejidad del régimen constituía un serio obstáculo para alumnos y docentes.

Las quejas de los alumnos se centraban principalmente en el accidentado proceso de inscripciones inter-semestrales que casi siempre les dejaba pocas opciones para escoger horarios cómodos o asignaturas de su interés, así como por la insatisfactoria calidad de las asesorías personalizadas. Los docentes por

37 Ministerio de Educación. "Resolución Nº 140: Regulaciones Complementarias sobre el proceso de evaluación en los niveles de Educación Preescolar, Básica, y Media Diversificada y Profesional”, Gaceta Oficial de la República de Venezuela, Caracas, 14 de abril de 1986.

38 Ministerio de Educación. "Resolución N 337: Reforma a la Resolución N 140 de fecha 9-4-86 que contiene las regulaciones complementarias sobre el proceso de evaluación en los niveles de Educación Preescolar, Básica, y Media Diversificada y Profesional", Gaceta Oficial de la República de Venezuela, Caracas, 14 de mayo de 1987.

39 El viernes negro de 1983 señala el ingreso formal de Venezuela al club de países endeudados y en recesión; características estas a las que el país había permanecido extraño debido fundamentalmente a la renta petrolera dinamizadora de toda la economía nacional. Algunos periodistas y analistas como Domingo Alberto Rangel incluso se atrevieron a llamar a este proceso de franco empobrecimiento, la "latinoamericanización" de Venezuela. Ver: Domingo Alberto Rangel. La Crisis Económica Mundial. (Caracas: Vadell Hermanos Editores, 1987).

40 Fermín, Momentos, 126. 
su parte, se quejaban de la poca claridad y practicidad de los procedimientos e instrumentos de evaluación. ${ }^{41}$ Sin embargo, el defecto más grave del régimen semestre-crédito era su incompatibilidad con la tendencia centralista de la administración pública venezolana y los altos costos de funcionamiento que significaba para una economía en franca recesión.

Y es que Venezuela asistía en esta época al triunfo definitivo del argumento tecnocrático de la Educación Rentable, esto es, del convencimiento casi unánime sobre la incapacidad del Estado para sufragar la totalidad de los gastos del Sistema Educativo; argumento que venía siendo esgrimido por los agentes gubernamentales desde 1964 y que para la víspera constituía una de las consignas principales de las comisiones reformistas designadas por el Estado venezolano. ${ }^{42}$

Sin embargo, las recomendaciones propuestas por las comisiones estatales retomar el proceso de descentralización iniciado con la regionalización de finales de los sesenta, al tiempo que se redujera drásticamente el gasto público- fueron tan arduamente combatidas por los grupos políticos tradicionales, esto es, firmes defensores del carácter centralista de la administración pública venezolana y de la utilización de la renta petrolera para mantener el consenso político, que fueron finalmente abandonadas, por lo menos en su primera versión. ${ }^{43}$

En efecto, frente a los discursos de regionalización y descentralización pronunciados por los voceros del Gobierno y publicados en planes, programas y proyectos; en la práctica, la administración del presidente Lusinchi llevó a cabo una revitalización y fortalecimiento del modelo centralista en Educación al minimizar y, en el caso del ensayo de régimen semestre-crédito eliminar, los proyectos modernizadores de la Reforma Educativa. ${ }^{44}$

El ensayo de régimen semestre-crédito fue relegado en la historia de la educación venezolana. Casi ninguna referencia a este experimento se encuentra reseñada en la literatura especializada y se recuerda como un episodio singular en la vida nacional, a pesar de haber constituido un esfuerzo considerable en recursos financieros, humanos y organizacionales, además de haber aplicado prácticas pedagógicas vanguardistas para su época.

\section{CONCLUSIÓN}

La Reforma Educativa aplicada en Venezuela a partir de 1969 formó parte de los procesos de reforma y transformación de las administraciones públicas de los Estados latinoamericanos durante la segunda mitad del siglo XX para adaptarse a los estándares internacionales promovidos por los organismos hemisféricos,

\footnotetext{
41 Ministerio de Educación, Informe de evaluación del régimen semestre-crédito, (Caracas: Ministerio de Educación, 1979).

42 Lenin Romero y Carlos Manterola, “Nueva Ley de Educación (Texto y comentario)”, Cuadernos de Educación, Número Extraordinario 78-79 (1980): 30 .

43 Manuel Peñalver, secretario general del partido Acción Democrática expresó lo que ha venido a considerarse el epitafio del proyecto COPRE: "Los venezolanos no somos suizos",

44 Rodríguez. Historia, 272.
} 
con el propósito de obtener financiamiento, asesoría técnica y capacitación especializada en el campo de la Educación.

Esta adaptación supuso la implantación de modelos educativos no tradicionales como el régimen semestral tanto en Educación Superior como en Educación Media, que además debía ser desarrollado en consonancia con el modelo de desarrollo regional promovido por la CEPAL para la diversificación geoeconómica.

En este sentido, el ensayo del régimen semestre-crédito supuso la profundización del currículo centrado en el desarrollo regional, con un pénsum de estudios dirigido al estudio de las potencialidades geoeconómicas regionales en estrecha colaboración con las fundaciones y corporaciones de desarrollo regional como FUDECO en la región centroccidental.

En Venezuela, no obstante las ventajas técnicas del mencionado régimen, la fragmentación de los planes de estudio en función de las necesidades y potencialidades de cada región, así como la variación en los tiempos de los procesos administrativos de cada plantel, devino en el abandono del ensayo llevado adelante en treinta y dos planteles durante más de diez años.

$\mathrm{Y}$ es que la fuerte tradición centralista del sistema político venezolano dio al traste con el plan de descentralización administrativa que suponía la regionalización del país, llevándose con ello los proyectos educativos que incorporaban diferenciaciones en los procedimientos administrativos de cada región.

Los factores arriba señalados deben enmarcarse además en los ciclos de bonanza y recesión de la economía rentista-petrolera venezolana. No es coincidencial que la cancelación del ensayo del régimen semestre-crédito ocurriera durante la crisis económica de la década de los ochenta, pues, en aras de hacer funcionar más eficientemente la administración del aparato escolar, el Estado impulsó una reforma curricular que recentralizó el Sistema Educativo, uniformando la gestión y reduciendo sus costos de mantenimiento.

En conclusión, el episodio del ensayo del régimen semestre-crédito ilustra, quizás como ningún otro proyecto de la Reforma Educativa, la dualidad entre el ideal de desarrollo nacional basado en la diversificación geoeconómica producto de la descentralización administrativa (incluida la administración escolar), y la práctica político-administrativa del centralismo como la forma más efectiva de gestionar la renta petrolera en todas los sectores de la vida pública nacional.

\section{FUENTES}

Ministerio de Educación. Memoria y Cuenta de 1975. Caracas: Ministerio de Educación, 1976.

Ministerio de Educación. Memoria y Cuenta de 1976. Caracas: Ministerio de Educación, 1977.

Ministerio de Educación, Guía del Estudiante. Régimen de Semestre-Crédito para los Institutos Experimentales de Educación Media Diversificada. Menciones de Humanidades y Ciencias. Caracas: Ministerio de Educación, 1975.

Ministerio de Educación, Ensayo Régimen Semestre Crédito: Una nueva estrategia y un nuevo enfoque para la educación diversificada. Caracas: Ministerio de Educación, 1977. 
Ministerio de Educación, Informe de evaluación del régimen semestre-crédito, Caracas: Ministerio de Educación, 1979.

Ministerio de Educación. "Resolución N 56 del 07 de abril de 1976", Gaceta Oficial de la República de Venezuela, Caracas, 22 de abril, 1976.

Ministerio de Educación. "Resolución N 12 del 05 de enero de 1977", Gaceta Oficial de la República de Venezuela, Caracas, 11 de enero, 1977.

Ministerio de Educación. “Resolución N 140: Regulaciones Complementarias sobre el proceso de evaluación en los niveles de Educación Preescolar, Básica, y Media Diversificada y Profesional", Gaceta Oficial de la República de Venezuela, Caracas, 14 de abril, 1986.

Ministerio de Educación. “Resolución N³37: Reforma a la Resolución Nº 140 de fecha 9-4-86 que contiene las regulaciones complementarias sobre el proceso de evaluación en los niveles de Educación Preescolar, Básica, y Media Diversificada y Profesional", Gaceta Oficial de la República de Venezuela, Caracas, 14 de mayo, 1987.

Presidencia de la República. “Decreto N 120: Reglamento para la Educación Secundaria y la Educación Técnica", Gaceta Oficial de la República de Venezuela, Caracas, 19 de agosto, 1969.

\section{REFERENCIAS}

Albornoz, Orlando. Sociedad y respuesta educativa. Caracas: Universidad Central de Venezuela. Ediciones de la Biblioteca, 1991.

Atcon, Rudolph. La universidad latinoamericana: Clave para un enfoque conjunto del desarrollo coordinado social, económico y educativo en América Latina. Bogotá: Unibiblos, 2005.

Brito Figueroa, Federico. Venezuela Contemporánea ipaís colonial? Caracas: Universidad Central de Venezuela. Ediciones de la Biblioteca, 1972.

Cardoso, Fernando Henrique y Faletto, Enzo. Dependencia y Desarrollo en América Latina. México: Siglo Veintiuno Editores, 1970.

Carnoy, Martin. La Educación como imperialismo cultural. México: Siglo XXI Editores, 1988.

Chacón, Alfredo. Cultura y Dependencia. Caracas: Monte Ávila Editores, 1975.

Di Tella, Torcuato S. (Supervisor). Diccionario de Ciencias Sociales y Politicas. Buenos Aires: PUNTOSUR Editores, 1989.

Fermín, Manuel. Momentos Históricos de la Educación Venezolana. Caracas: Editorial Romor, 1989.

Fernández Heres, Rafael. Regionalización de la Educación en Venezuela. Caracas: Instituto Nacional de Cooperación Educativa, 1982.

Germani, Gino. Política y Sociedad en una época de transición: De la sociedad tradicional a la sociedad de masas. Buenos Aires: Editorial Paidós, Buenos Aires, 1962.

Graffe, Gilberto José. Políticas Públicas Educativas frente a la Crisis, Reforma del Estado y modernización de la Educación Venezolana. Universidad Central de Venezuela, Ediciones Secretaría de la UCV, Ediciones Vicerrectorado Académico, 2005.

Guerra C., Alexis J. La Planificación en la Región Centro Occidental 1958-2005. Barquisimeto: Universidad Centroccidental Lisandro Alvarado, Cátedra Libre Banco Central de Venezuela, 2012.

Jaguaribe, Helio y otros. La dependencia político-económica de América Latina. México: Siglo XXI Editores, 1978.

Malavé Mata, Héctor. Formación Histórica del Antidesarrollo de Venezuela. Caracas: Ediciones Rocinante, 1975.

Márquez, Trino. El Estado social de derecho. Origen, evolución y situación actual. Caracas: Editorial PANAPO, 1998.

Maza Zavala, D. F. Los mecanismos de la dependencia. Caracas: Ediciones Rocinante, Fondo Editorial Salvador de la Plaza, 1973.

Medina, Ramón Daniel. La Pedagogía Tecnocrática a la Luz del Pensamiento Pedagógico Universal. Maracaibo: Ediciones de la Universidad del Zulia, 2005.

Mora García, José Pascual. Del Fin de la Historia a la Postmodernidad. S/L: Universidad de Los Andes, Vice-Rectorado de Extensión Universitaria, Núcleo Universitario del Táchira, 1996. 
Muñoz L., Carlos A. El Estado Venezolano y su política regional. Mérida: Textos de la Universidad de Los Andes. Facultad de Ciencias Forestales, 1990.

Parsons, Talcott. Estructura y procesos en las sociedades modernas. Madrid: Instituto de Estudios Políticos, 1966.

Prebisch, Raúl. Hacia una dinámica del desarrollo latinoamericano. Uruguay: Colección Cuadernos de América. Ediciones de la Banda Oriental, 1967.

Prieto Figueroa, Luis Beltrán. El Estado y la Educación en América Latina. Caracas: Colección Estudios. Monte Ávila Editores, 1978.

Rangel, Domingo Alberto. La Crisis Económica Mundial. Valencia: Universidad de Los Andes, Vadell Hermanos Editores, 1987.

Rodríguez, Nacarid (Compiladora.). Historia de la Educación Venezolana. Seis Ensayos. Caracas: Universidad Central de Venezuela, Facultad de Humanidades y Educación, Comisión de Estudios de Postgrado, 1996.

Rojas, Reinaldo. Universidad Centroccidental Lisandro Alvarado: Una Historia de 50 años. Barquisimeto: Universidad Centroccidental Lisandro Alvarado, Ediciones del Rectorado, 2012.

Sunkel, Oswaldo. El subdesarrollo latinoamericano y la teoría del desarrollo. México: Siglo XXI Editores, 1970.

\begin{tabular}{l|l|} 
& \\
\hline Rodríguez Suárez, Jadhiel Alejandro. “Desarrollo y Reforma \\
Educativa en Venezuela: El ensayo del Régimen semestre-crédito \\
en educación media (1976-1986)" Revista Historia de la Educación \\
Latinoamericana. Vol. 20 No. 30 (2018): 243-259.
\end{tabular}

